\title{
Evidence for Strong Inversed Shear of Toroidal Rotation at the Edge Transport Barrier in ASDEX Upgrade
}

\author{
T Pütterich, ${ }^{*}$ E. Wolfrum, R. Dux, C.F. Maggi, and ASDEX Upgrade Team \\ Max-Planck-Institut für Plasmaphysik, EURATOM Association, D-85748 Garching, Germany
}

\begin{abstract}
The toroidal rotation of H-mode plasmas in ASDEX Upgrade is studied in the outermost $5 \mathrm{~cm}$ of the confined plasma. The projection of the rotation velocity along the line of sight (approx. toroidal) is measured using charge exchange recombination spectroscopy, with a radial resolution of up to $3 \mathrm{~mm}$ and a temporal resolution of $1.9 \mathrm{~ms}$. At about $1 \mathrm{~cm}$ inside the separatrix the rotation exhibits a local minimum. From there, the rotation in co-direction increases towards the plasma center and towards the separatrix. The latter increase is the focus of this work. It is situated in the region of the edge transport barrier and amounts to $10-20 \mathrm{~km} / \mathrm{s}$. It is observed for $\mathrm{D}^{+}, \mathrm{He}^{2+}, \mathrm{B}^{5+}$, and $\mathrm{C}^{6+}$. The described rotation feature at the edge is not visible during an ELM crash and is probably connected to the occurrence of steep gradients in this plasma region.
\end{abstract}

\section{INTRODUCTION}

In H-mode fusion plasmas [1], an edge transport barrier (ETB) [1] is found leading to steep gradients in the plasma parameters at the last few $\mathrm{cm}$ of the magnetically confined plasma. The ETB is disturbed frequently by edge localized modes (ELMs, here: type-I), which are characterised by bursts of particles and energy being expelled from the plasma edge. In ASDEX Upgrade [2] the duration of an ELM is in the range of a millisecond, while the time between ELMs can be as long as tens of milliseconds. The width of the ETB region and the gradients cannot be predicted by a model using first principles. Nevertheless, the performance of ITER and a future fusion reactor is very sensitive to the densities and temperatures at the top of the edge pedestal. It is widely assumed that the occurrence of a sheared plasma flow perpendicular to the magnetic field suppresses turbulence and thus leads to reduced transport, i.e the ETB. The persistence of the ETB can be understood by the existence of a self-consistent radial electric field maintaining a sheared $\mathrm{E} \times \mathrm{B}$ flow, while being produced by the gradients and the plasma flow. An open issue still is the influence of the toroidal rotation profile on the ETB and the $\mathrm{H}$ mode transition and vice versa the effects of the H-mode edge on the toroidal rotation (e.g. [3]). Measurements focusing on the pedestal need to provide high radial resolution $(<1 \mathrm{~cm})$ and fast temporal resolution $(\approx 1 \mathrm{~ms})$ due to the frequent occurrence of ELMs. Diagnostic systems capable of measuring highly resolved edge profiles are scarce. Additionally, the rotation measurements need to be spatially related to electron temperature and density measurements to enable a comprehensive localization of the separatrix relative to the rotation measurements.

\section{EXPERIMENT}

The viewing geometry of the edge charge exchange recombination spectroscopy (CXRS, [4]) system at ASDEX Upgrade is adjusted to high spatial resolution. The f/4 optics gathers the light, which is transmitted by $400 \mu \mathrm{m}$ optical fibres and analyzed by a f/4 spectrometer employing commer- cial objective lenses with a focal length of $280 \mathrm{~mm}$ and a grating with 2400 grooves $/ \mathrm{mm}$. The dispersed light is detected by a CCD camera with electron multiplication readout allowing for a pixel rate of $10 \mathrm{MHz}$ at acceptable readout noise. The light from eight radial positions at the outboard midplane (separated by $\approx 1 \mathrm{~cm}$ ) is analyzed by the spectrometer with a repetition time of $1.9 \mathrm{~ms}$. The evaluation of spectral lines emitted following a charge exchange $(\mathrm{CX})$ reaction is used to obtain local information about the ion temperature $T_{i}$ and toroidal rotation $\mathrm{v}_{\mathrm{tor}}$, which are evaluated by Doppler broadening and line shift. The systematical uncertainties of the rotation measurements originate from uncertainties in the wavelength calibration of the spectra. These have been thoroughly quantified by measurements on calibration lamps and the analysis of intrinsic impurity lines. The absolute rotation velocities are uncertain by $5 \mathrm{~km} / \mathrm{s}$, while the relative uncertainties between different LOS are less then $1-2 \mathrm{~km} / \mathrm{s}$. Statistical uncertainties are indicated in the following figures using vertical, symmetric error bars. The radial resolution of the setup is determined by the size of the plasma volume (diameter of $4 \mathrm{~mm}$ ) which is imaged onto the optical fibres. Additionally, the measurements are smeared by $\approx 5 \mathrm{~mm}$ due to the fact, that the plasma is curved within the beam width of $0.2 \mathrm{~m}$. However, for emissions, which exhibit a strong radial gradient in intensity, the spatial resolution is strongly enhanced, reaching for the presented cases values down to $3 \mathrm{~mm}$. With this new diagnostic, plasma discharges in H-mode have been studied, while the fast temporal resolution was used to analyse the development of the measured quantities within an ELM cycle. To obtain best data, the plasma discharges were horizontally swept by $2 \mathrm{~cm}$, such that the plasma was not disturbed while the edge of the plasma moved through the view of the described LOS. The measured spectra are then analysed by a spectral line fit. Measurements have been obtained for $\mathrm{C}^{6+}, \mathrm{B}^{5+}, \mathrm{He}^{2+}$ and $\mathrm{D}^{+}$ using the CX spectral lines at $529.059 \mathrm{~nm}(8-7), 494.497 \mathrm{~nm}$ (7-6), $467.857 \mathrm{~nm}$ (4-3) and $656.103 \mathrm{~nm}$ (3-2), respectively. In the following, data are presented which result from a fit with two Gaussians taking the contribution of passive line emissions into account, while for the comparison between different species beam modulation was applied, i.e. the heating beam, which is used for the CX measurement, is switched on and off 
while another beam is switched off and on to compensate the total heating power. Using this latter method the background emissions are subtracted and a single Gauss fit can be used to obtain the parameters of the CX emission.

\section{RESULTS}

Figure 1 depicts the fit results, i.e. the CX-line intensity, $T_{i}$ and $\mathrm{v}_{\text {tor }}$ for an H-mode discharge (\#22273) with type-I ELMs. In this discharge, one neutral beam source $\left(\mathrm{P}_{\mathrm{NBI}}=2.5 \mathrm{MW}\right)$, electron cyclotron heating $\left(\mathrm{P}_{\mathrm{ECH}}=1.4 \mathrm{MW}\right)$ and ohmic heating $\left(\mathrm{P}_{\mathrm{OH}}=0.4 \mathrm{MW}\right)$ was used to obtain $\mathrm{H}$-mode at a line integrated electron density $\mathrm{n}_{\mathrm{e}}$ of about $8 \cdot 10^{19} \mathrm{~m}^{-3}$, plasma current $I_{p}$ of $1 \mathrm{MA}$ and a magnetic field $\mathrm{B}_{\mathrm{t}}$ of $2.4 \mathrm{~T}$ resulting in an ELM-frequency of about $65 \mathrm{~Hz}$. The temporal resolution of $1.9 \mathrm{~ms}$ allows us to synchronize the data to the occurrence of an ELM. In figure 1 four columns are presented labelled with '-7 ms', '-1 ms', '+1 ms' and ' +5 ms' denoting the relative time to the following or preceding ELM at which the measurements are taken, while light is included $\pm 1.45 \mathrm{~ms}$ around these time points. The plasma radius of the measurement location is determined by a numerical integration along the LOS which determines the CX emission intensity in the observed plasma volumes. The alignment to the $\mathrm{n}_{\mathrm{e}}$ and $\mathrm{T}_{\mathrm{e}}$ profiles and the magnetic measurements is discussed below. The effects of the ELM can be studied for all quantities, but will play a minor role for this work. For the $\mathrm{v}_{\text {tor }}$-profile, a feature is visible in between ELMs. The $\mathrm{v}_{\text {tor }}$-profile exhibits a local minimum at $1 \mathrm{~cm}$ inside the separatrix. On the last centimeter of the confined plasma $v_{\text {tor }}$ increases by about $10-20 \mathrm{~km} / \mathrm{s}$ reaching about $25 \mathrm{~km} / \mathrm{s}$ at the separatrix. This feature is visible during the whole ELM cycle only interrupted for about 2-3 ms by the ELM. The behaviour during the ELMs cannot be judged by the measurements, because the exposure time of $1.9 \mathrm{~ms}$ is too long. Still, it is remarkable, that in figure 1 no data points indicate the dip in rotation velocity for the time labelled ' $+1 \mathrm{~ms}$ '. In all other frames the dip is clearly visible and also for the time frame ' $+3 \mathrm{~ms}$ ' (omitted in figure 1) a dip is visible.

For all studied plasma discharges in $\mathrm{H}$-mode, this rotation feature is visible to some extent, while in L-mode it has not been observed. The optimized radial alignment is presented in figure 2. The uncertainty of the separatrix position from magnetic reconstruction can be greatly reduced with the help of the steep $\mathrm{T}_{\mathrm{e}}$-profiles. By setting $\mathrm{T}_{\mathrm{e}}$ at the separatrix to $\approx 100 \mathrm{eV}$, a mandatory condition set by the parallel heat transport and power balance [5] is satisfied. The additional $n_{e^{-}}$ profiles from the Li-beam are thereafter aligned to the $n_{e^{-}}$ measurements of the Thomson scattering, as for the latter diagnostic the measurement volumes are identical for electron temperatures and densities. The CX-data are then aligned with an accuracy of about 2-3 mm, such that the steep $T_{i}$-gradient region coincides with that of the $\mathrm{T}_{\mathrm{e}}$-measurement (see figure 2 ). The necessary corrections are typically smaller than $5 \mathrm{~mm}$. With all profiles aligned it is easily visible that the inversion of the rotation shear is located directly at the region with steep

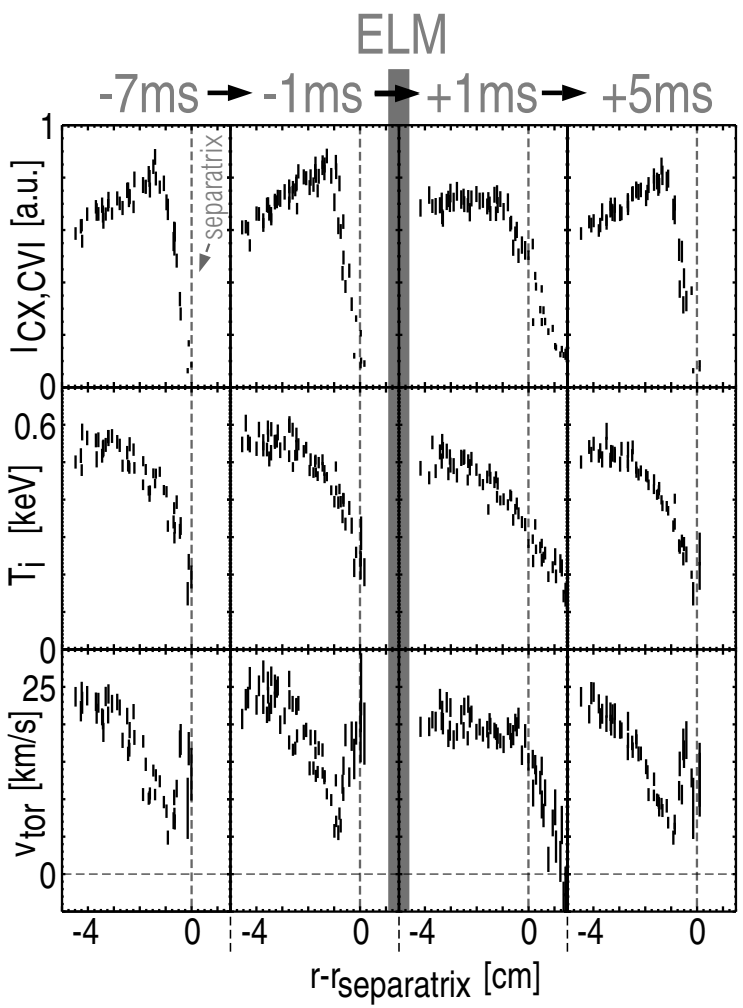

FIG. 1: Measurements derived from the active CX-component between beam neutrals and fully-stripped $\mathrm{C}$. The data were obtained from a $300 \mathrm{~ms}$ long phase in which the plasma was horizontally swept by $2 \mathrm{~cm}$. The intensity of the CX-component $\left(\mathrm{I}_{\mathrm{CX}, \mathrm{CVI}}\right), \mathrm{T}_{\mathrm{i}}$ and the $\mathrm{v}_{\text {tor }}$ (positive is in co-direction) of the plasma are depicted in different phases of an ELM-cycle.

gradients in the $\mathrm{n}_{\mathrm{e}^{-}}, \mathrm{T}_{\mathrm{e}^{-}}$and $\mathrm{T}_{\mathrm{i}^{-} \text {-gradients. }}$

Taking the depicted plasma parameters into account, it is derived that the collisionality of $\mathrm{C}$ is above the PfirschSchlüter limit, that of $\mathrm{He}$ is about at the limit, while that of deuterium (D) is below. This poses a motivation to investigate the feature in $v_{t o r}$ for different impurities and D. Such measurements have been performed in the discharges for which data are presented in figure 3 . The upper measurements in figure $3\left(\mathrm{D}^{+}, \mathrm{B}^{5+}\right)$ originate from discharges with $\mathrm{I}_{\mathrm{p}}=1.1 \mathrm{MA}$, $\mathrm{B}_{\mathrm{t}}=2.75 \mathrm{~T}, \mathrm{n}_{\mathrm{e}}=7.8 \cdot 10^{19} \mathrm{~m}^{-3}, \mathrm{P}_{\mathrm{NBI}}=2.5 \mathrm{MW}$ and $\mathrm{P}_{\mathrm{ECH}}=0.75 \mathrm{MW}$. The discharge parameters of the lower measurements in figure $3\left(\mathrm{He}^{2+}, \mathrm{B}^{5+}, \mathrm{C}^{6+}\right)$ are $\mathrm{I}_{\mathrm{p}}=1 \mathrm{MA}$, $\mathrm{B}_{\mathrm{t}}=2.5 \mathrm{~T}, \mathrm{n}_{\mathrm{e}}=7.5 \cdot 10^{19} \mathrm{~m}^{-3}, \mathrm{P}_{\mathrm{NBI}}=5 \mathrm{MW}$ and $\mathrm{P}_{\mathrm{ECH}}=$ 0.6 MW. All measurements originate from time points not disturbed by ELMs. The measurements on $\mathrm{C}$ exhibit larger error bars, because the $\mathrm{C}$ concentration for the discharge series has been below $0.2 \%$ due to a recent boronization. However, from the experience in various other discharges it is observed that there is no significant difference between the rotation velocities of $\mathrm{B}^{5+}$ and $\mathrm{C}^{6+}$. It must be noted, that for $\mathrm{D}$ and $\mathrm{He}$ the beam halo and plume [4] effect have not been corrected for. Both effects lead to a smoothing of steep gradients in the measured rotation. While the plume effect of He is estimated to 


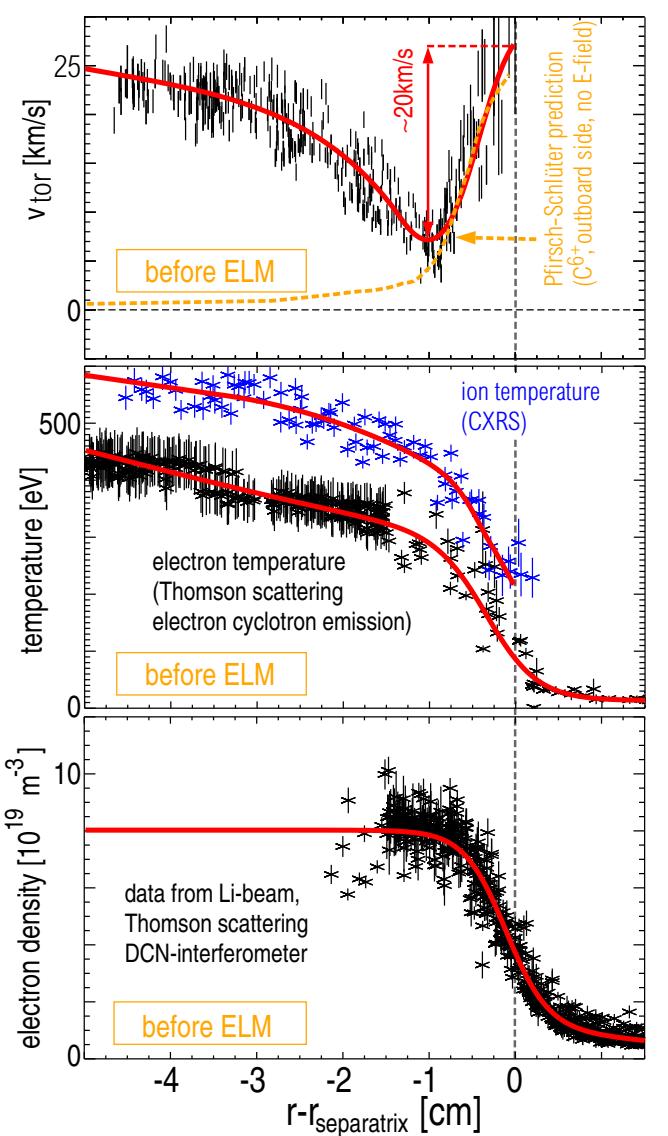

FIG. 2: Measurements of $\mathrm{v}_{\text {tor }}\left(\mathrm{C}^{6+}\right.$, positive is in co-direction) in discharge \#22273 (H-mode) overlaying all data from $10 \mathrm{~ms}$ before the ELM up to the ELM. Below $\mathrm{T}_{\mathrm{i}}\left(\mathrm{C}^{6+}\right), \mathrm{T}_{\mathrm{e}}$ and $\mathrm{n}_{\mathrm{e}}$ profiles are depicted for corresponding times. The alignment of the profiles with respect to the separatrix is described in the text. The lines are introduced to guide the eye.

play no major role in the measurements, the halo effect for D is estimated to mix the measurement on a radial scale of about 10-20 mm depending on the local plasma parameters. This estimation is based on how far a neutral cloud (born by CX with the beam) would expand until being ionized by the electrons.

\section{DISCUSSION}

The measurements have been thoroughly verified by comparisons of evaluation methods and by measurements using different impurities, hence spectral regions. From the DIIID tokamak measurements are presented in [6] which indicate a similar feature at the plasma edge, however, it is not discussed nor verified and the exact alignment to the $n_{e}, T_{e}$ profiles and the resolution of the feature are not performed in [6]. An exact knowledge of the separatrix position is of high importance for validating and aligning the measurements with respect to other diagnostics and for narrowing down possible explanations. Various effects which could lead to a rotation
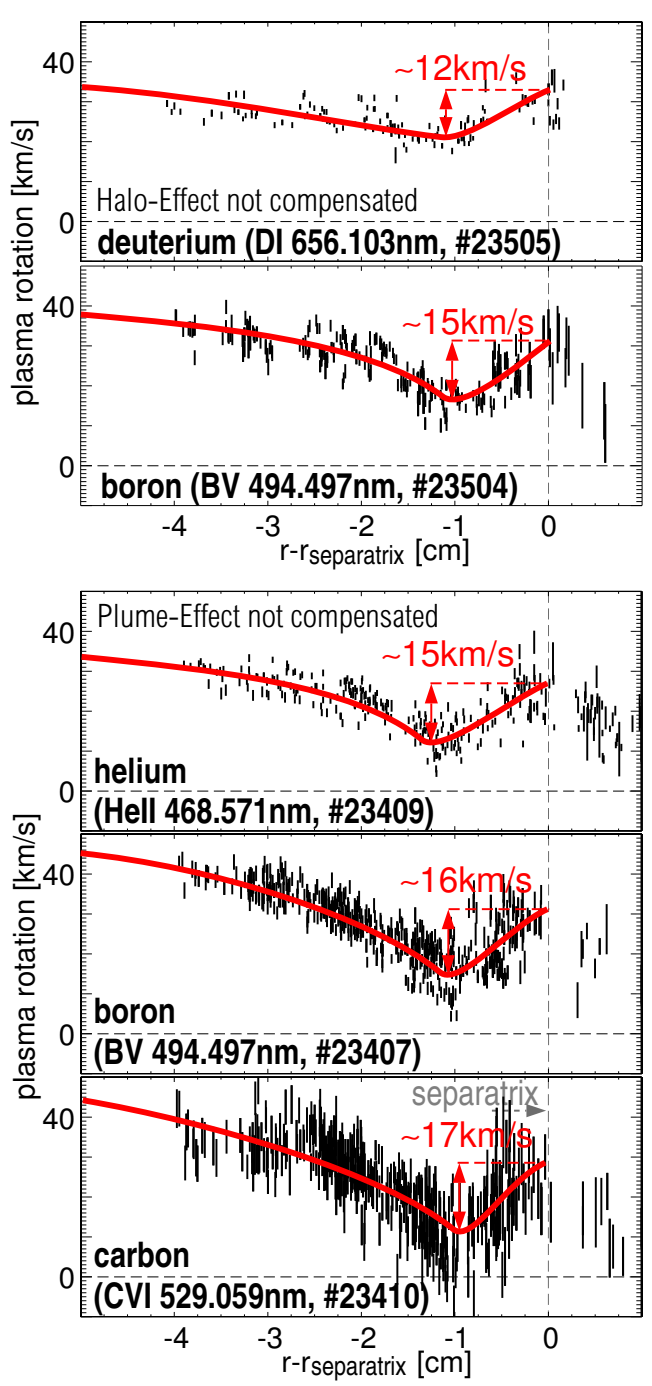

FIG. 3: Measured $\mathrm{v}_{\text {tor }}$ of different species for similar discharges. The measurements of $\mathrm{D}$ and He have not been corrected for the halo and plume effect. The latter lead to an apparent flattening of gradients in $\mathrm{v}_{\text {tor }}$.

feature as described above are discussed in the following. The perpendicular velocity $v_{\perp, j}$ of a particular charge state of an species is fixed via the radial force balance which we denote as $v_{\perp, j} B_{\|}=\frac{\partial p_{j}}{\partial r n_{j} q_{j}}-\frac{\partial \Phi}{\partial r}$ where $p_{j}$ is the partial pressure, $n_{j}$ the density and $q_{j}$ the charge of the considered species and $\Phi$ is the electric potential. The assumption is made that $p_{j}$ and $\Phi$ are constants on a flux surface. For zero electric field $v_{\perp, j}$ equals the diamagnetic velocity $v_{d i a, j}$ which arises from the pressure gradient. For the presented cases the $v_{d i a}$ of $D^{+}$are in the range of $20-25 \mathrm{~km} / \mathrm{s}$ at the radial position of the steep gradients. The projection onto the LOS of the measurements is less than $1 / 8$ (i.e. $<3 \mathrm{~km} / \mathrm{s}$ in co-direction) of that value. It is known from the measurements in \#22273 (s. figure 1) [7] that the pressure gradient of $C^{6+}$ is $\approx 2-3$ times steeper than that of $D^{+}$, which is in agreement with predictions of neoclassical theory [7]. Due to the difference in $q_{j}$ the dia- 
magnetic velocity of $D^{+}$is about 2-3 times larger than that of $C^{6+}$. An electric field is typically predicted to be negative inside the separatrix, which would make $v_{\perp, j}$ smaller and which would leave the absolute difference between different impurity species unchanged. So, the projection of $v_{\perp, j}$ is too small to explain the observations. However, $v_{\perp, j}$ is not divergence free and a parallel flow (Pfirsch-Schlüter PS) is necessary to provide zero divergence - a strict boundary condition - for the plasma flow. These parallel flows have been determined for $D^{+}$and $C^{6+}$ using the measured pressure profile (different for $\mathrm{D}^{+}$and $\mathrm{C}^{6+}$ ) and assuming zero electric field. A strong increase of the parallel velocity is predicted in codirection at the outboard side and in counter direction at the inboard side such that the flux average parallel velocity is only a few $\mathrm{km} / \mathrm{s}$, while locally just inside of the separatrix at the outboard side parallel velocities of up to $70 \mathrm{~km} / \mathrm{s}$ and $25 \mathrm{~km} / \mathrm{s}$ are predicted for $D^{+}$and $C^{6+}$ (for latter see figure 2), respectively. In the presence of a negative electric field the perpendicular velocity is smaller than the purely diagmagnetic velocity and thus the necessary PS flows in parallel direction are also reduced. For a typical field (like measured in $[8,9]$ ) in the range of $30 \mathrm{kV} / \mathrm{m}$ the PS flows at the outboard side reduce to about $40 \mathrm{~km} / \mathrm{s}$ and $-5 \mathrm{~km} / \mathrm{s}$ for $D^{+}$and $C^{6+}$, respectively. It should be noted that the electric field is obtained by Doppler reflectometry which results are not influenced by the discovered feature in $\mathrm{v}_{\text {tor }}$ (cf. [9]). The PS flow is in principle of the right order of magnitude and exhibits a radial shape which looks promising for explaining the observations (s. figure 2), however, the predicted difference between various species (e.g. $D^{+}$and $C^{6+}$ ) is not observed in experiment.

Additional differences of the rotation velocity for different species could be expected from neoclassical effects. These have been evaluated by the code NEOART [10]. NEOART calculates the collisional transport coefficients for an arbitrary number of impurities including collisions between all components. The code solves the set of linear coupled equations for the parallel velocities in arbitrary toroidally symmetric geometry for all collision regimes. The classical fluxes are given by Eqs.(5.9) and (5.10) in [11]. The equations for the banana plateau contribution are equal to that used in [12]. The Pfirsch-Schlüter contribution is calculated from the coupled equations (6.1-2) and (6.14-15) in [11], as described in [13]. From the code the differences in rotation between D and the impurities are predicted for the measured input profiles of $n_{e}$, $T_{e}, T_{i}$ and impurity concentrations, which were estimated using typical values. The resulting differences are in the range of $2 \mathrm{~km} / \mathrm{s}$ and cannot be resolved experimentally. It must be noted that approaching the separatrix the neoclassical approximation breaks down for $\mathrm{D}^{+}$, because the poloidal gyroradius gets comparable to the gradients in the plasma parameters.

In experiment, plasma flows pointing in co-direction have been observed in the scrape-off layer (SOL) of ASDEX Upgrade exhibiting Mach numbers of about 0.6 just outside the separatrix [14]. This corresponds to $\approx 40 \mathrm{~km} / \mathrm{s}$ at $\mathrm{T}_{\mathrm{e}}=\mathrm{T}_{\mathrm{i}}=$ $50 \mathrm{eV}$. Due to viscous forces such rotation in the SOL could yield a boundary condition to the rotation of the plasma inside the separatrix, while the electric field gradient and rotation would adjust self-consistently. Into the same direction hints the fact, that the banana width of thermal D is at the separatrix about $8 \mathrm{~mm}$ wide, which corresponds about to the width of the feature in $\mathrm{v}_{\text {tor }}$. This calls for detailed fluid simulations taking viscosity and drift velocities (also in the SOL) selfconsistently into account. In [15], this has been done and similar structures as observed have been obtained from modelling. It should be noted that in the SOL there is a positive, radial electric field outside the separatrix providing a perpendicular plasma rotation with a toroidal projection in the co-direction. The magnitude of this projection is too small to contribute directly to the measurement, but its correlated Pfirsch Schlüter flow is suspected to be responsible for the SOL flows.

Independently, the feature might play a role for the momentum transport of the plasma, for which the flux surface averaged momentum is important. If the flow is PS-like, a flow at the inboard side of the tokamak would exist which makes the flux surface average small. However, if PS does not apply, a minimum in the flux surface averaged toroidal momentum implies a momentum sink or peculiar momentum transport. Further measurements on the inboard side are needed to investigate this question.

* Electronic address: Thomas.Puetterich@ipp.mpg.de

[1] F. Wagner et al., Phys. Rev. Lett. 49, 1408 (1982).

[2] A. Herrmann and O. Gruber, Fusion Science and Technology 44, 569 (2003).

[3] J. Rice et al., Nuclear Fusion 47, 1618 (2007).

[4] R. J. Fonck, D. S. Darrow, and K. P. Jaehnig, Physical Review A 29, 3288 (1984).

[5] J. Neuhauser et al., Plasma Physics and Controlled Fusion 44, 855 (2002).

[6] J. S. deGrassie et al., Physics of Plasmas 14, 056115 (2007).

[7] T. Pütterich et al., Europhysics Conference Abstracts (CDROM, Proc. of the 35rd EPS Conference on Plasma Physics) EPS, Geneva, 2008, Vol. 32F, pp. P-2.083.

[8] J. Schirmer et al., Nuclear Fusion 46, S780 (2006).

[9] G. D. Conway et al., 8th International Reflectometry Workshop (IRW8, St. Petersburg, 2007).

[10] A. G. Peeters, provided the NEOART code, 1998 .

[11] S. P. Hirshman and D. J. Sigmar, Nuclear Fusion 21, 1079 (1981).

[12] W. A. Houlberg, K. C. Shaing, S. P. Hirshman, and M. C. Zarnstorff, Physics of Plasmas 4, 3230 (1997).

[13] A. G. Peeters, Physics of Plasmas 7, 268 (2000).

[14] H. W. Müller et al., Journal of Nuclear Materials 337-365, 605 (2007).

[15] V. Rozhansky et al., Plasma Physics and Controlled Fusion 48, 1425 (2006). 